PESQUIMAT, Revista de la F.C.M. de la

Universidad Nacional Mayor de San Marcos

Vol. XVII N¹, pp. 25-37, Lima - Perú, Abril 2014

\title{
RELACIÓN ENTRE LA INTELIGENCIA EMOCIONAL Y LAS ESTRATEGIAS DE APRENDIZAJE EN ESTUDIANTES DE LA FACULTAD DE CIENCIAS MATEMÁTICAS
}

\author{
Carlos Alberto Peña Miranda ${ }^{1}, \quad$ Elizabeth Cosi Cruz ${ }^{2}$
}

Resumen: El objetivo del presente trabajo de investigación fue determinar si la inteligencia emocional y las estrategias cognitivas se relacionan entre si en una muestra de 260 alumnos del segundo, cuarto, sexto y octavo ciclo de la Facultad de Ciencias Matemáticas, de ambos sexos, cuyas edades fluctúan entre 18 y 31 años de edad. Para establecer la relación entre dichas variables se utilizó el diseño correlacional; en tanto para la recolección de datos, se utilizó el cuestionario de coeficiente emocional elaborado por BarOn y el inventario de estrategias de aprendizaje elaborado por Weinstein y Colbs, cuya validez se estableció mediante el análisis factorial exploratorio y la confiabilidad a través del análisis interno donde se obtuvo un alfa de Cronbach de 0,96 para la prueba de inteligencia emocional y de 0,91 para inventario de estrategias de aprendizaje. Los resultados estadísticos obtenidos nos muestran que la inteligencia emocional se encuentra relacionada significativamente a las estrategias de aprendizaje de los alumnos de la muestra $(\mathrm{r}=$ 0,83), así mismo se encontró que los alumnos varones y mujeres de los diversos ciclos académicos, básicamente, presentan los mismos niveles de inteligencia emocional y de estrategias de aprendizaje.

Palabras claves: Inteligencia emocional. Estrategias de aprendizaje. Estrategias metacognitivas.

\section{RELATION BETWEEN THE EMOTIONAL INTELLIGENCE AND THE LEARNING STRATEGIES IN STUDENTS OF THE FACULTY OF MATHEMATICAL SCIENCES}

\footnotetext{
Abstract: The objective of this present research work was to determine whether emotional intelligence and cognitive strategies relate to each other in a sample

${ }^{1}$ UNMSM, Facultad de Ciencias Matemáticas, e-mail: cpenam@unmsm.edu.pe

${ }^{2}$ UNFV, Facultad de Educación, e-mail: ecosi_euded@yahoo.es
} 
of 260 students in second, fourth, sixth and eighth cycle of the Faculty of Mathematical Sciences, of both sexes, ages 18 to 31 years of age. To establish the relationship between these variables using correlational; while for data collection, it was used the emotional coefficient questionnaire made by BarOn and the Learning strategies Inventory made by Weinstein and Colbs, whose validity was established by exploratory factor analysis and reliability through internal analysis which yielded a Cronbach alpha of 0,96 was obtained for the test of emotional Intelligence and 0,91 for learning strategies inventory. The statistical results obtained show that emotional intelligence is significantly related to learning strategies of students in the sample $(\mathrm{r}=$ $0,83)$, also found that male and female students of various academic cycles, basically, have the same levels of emotional intelligence and learning strategies.

Key words: Emotional intelligence. Learning strategies. Metacognitive strategies.

\section{Introducción}

La educación emocional surge como una respuesta educativa a una serie de necesidades que se dan en la sociedad actual: ansiedad, depresión, problemas de disciplina, violencia, drogadicción, trastornos de la alimentación, etc. Investigaciones recientes sobre esta temática han demostrado cómo el "analfabetismo emocional" tiene efectos muy negativos sobre las personas y sobre la sociedad (Goleman, 1996).

La importancia del control y la regulación de las emociones surgen, también, de la necesidad que tiene el ser humano de no dejarse llevar por los impulsos emocionales, ya que lo contrario tendría consecuencias muy negativas tanto a nivel personal como social. Además, todas las personas necesitan sentirse seguras emocionalmente, es decir, tener sentimientos de bienestar y estabilidad emocional. El factor emocional se considera como un elemento muy importante en la prevención y/o desarrollo de algunas enfermedades: se está demostrando que emociones negativas como la ira, el miedo, el estrés, la depresión, etc. tienen un efecto directo sobre la salud (Perea, 2002).

Por otra parte el impacto de la inteligencia emocional en el desarrollo académico de los alumnos no siempre ha sido valorado en sus justos términos. En este sentido, se debe tener en cuenta que los factores emocionales vinculados con el sentimiento de los alumnos son fundamentales. A menudo se llevan a cabo planes para mejorar el rendimiento académico de los alumnos, pero en ellos se obvia muchas veces el aspecto emocional, cuando éste tiene una gran importancia en el 
comportamiento académico de los alumnos.

Según Barios, las estrategias de aprendizaje constituyen uno de los focos de investigación más relevante en lo que se refiere a materia educativa. Éstas sirven como herramientas que facilitan la adquisición, desarrollo y puesta en marcha de procesos que permiten adquirir contenidos, facilitando un proceso de aprendizaje eficaz.

Las estrategias suponen, a su vez, un cambio de paradigma, desde el conductista basado en la relación estímulo-respuesta, a otro que nace del enfoque constructivista, caracterizado por la influencia de variables mediadoras y por la elaboración activa de significados por parte del propio alumno.(Barios, s.f)

Según Bara, con el objetivo de que el alumno sea un sujeto activo en el proceso de aprender, resulta de especial utilidad la enseñanza de estrategias de aprendizaje, las cuales ayudan a planificar, regular y evaluar el aprendizaje.

El objetivo de la presente investigación es determinar la relación que existe entre la inteligencia emocional y las estrategias de aprendizaje en los alumnos de la Facultad de Ciencias Matemáticas. Esto es muy importante por que aportará datos que puedan ayudarnos a orientar de mejor manera el proceso de formación profesional de los alumnos de la Facultad de Ciencias Matemáticas en tanto son ellos futuros profesionales destinados a liderar todo lo relacionado a esta área del conocimiento. Por otro lado, la presente investigación buscó desarrollar un conjunto de recomendaciones destinadas a establecer programas de desarrollo y/o recuperación de la inteligencia Emocional y estrategias de aprendizaje en los alumnos de la Facultad de Ciencias Matemáticas.

\section{Metodología}

En la medida que las variables independientes ya han ocurrido y por lo tanto no pueden ser manipuladas por el investigador, el presente estudio es una investigación de tipo no experimental. Para la selección del diseño de investigación se ha utilizado como base el libro de Hernández, R., Fernández, C. y Baptista, P. (2006) titulado "Metodología de la Investigación". Según estos autores el diseño adecuado para esta investigación es de tipo transversal correlacional.

En nuestro caso, pretendemos relacionar la inteligencia emocional y las estrategias de aprendizaje en alumnos de la Facultad de Ciencias Matemáticas. 
La muestra está conformada por 67 alumnos del segundo ciclo, 75 alumnos del cuarto ciclo, 69 alumnos del sexto ciclo y 49 alumnos del octavo ciclo, lo que hace un total de 260 alumnos con edades que fluctúan entre 18 y 31 años de edad, siendo las de mayor porcentaje las edades de 20 (19,6\%), 21 años $(18,1 \%)$ y 22 años $(13,1 \%)$.

La aplicación de los instrumentos se realizó durante el semestre académico 2013 - II del 15 de agosto al 20 de setiembre del 2013 y se utilizó los siguientes instrumentos:

1) El Inventario de Coeficiente Emocional de BarOn - ICE (BarOn, 1997) incluye 133 ítems cortos y emplea un grupo de respuestas de cinco puntos. Se requiere aproximadamente de 30 a 40 minutos para completarlo; sin embargo, no existen límites de tiempo impuestos. El ICE de BarOn es adecuado para individuos de 16 años o más. La evaluación genera los resultados de tres escalas de validez, un resultado CE total, resultados de cinco escalas compuestas y los resultados de quince subescalas del CE.

2) La prueba de Inventario de Habilidades y Estrategias de Aprendizaje elaborado por Weinstein y Colbs, es un instrumento de evaluación diseñado para medir el grado en el que los alumnos usan métodos y estrategias de estudio y aprendizaje y consiste en un cuestionario de 77 enunciados. En consecuencia sirve para recolectar información acerca de las prácticas de estudio y aprendizaje.

\section{Resultados y Discusión}

De acuerdo a los resultados del análisis psicométrico de la prueba de Inteligencia emocional, el instrumento es confiable en la medida que obtuvo un coeficiente alfa de Cronbach que llega a 0,96, lo que indica que la prueba es confiable. En lo que se refiere a la validez, se realizó a través del Análisis Factorial Exploratorio, aplicando el método de los componentes principales, la que permite observar que la prueba de Inteligencia emocional presenta una Medida de Adecuación del Muestreo de Kaiser-Meyer-Olkin que asciende a 0,69; el cual puede clasificarse como adecuado. Además presenta un test de esfericidad de Bartlett cuyo valor denota que la matriz de correlaciones entre áreas es significativa, la varianza explicada alcanza un nivel de $81,09 \%$. Estos hallazgos indican que la prueba tiene validez de constructo. Los resultados muestran que la prueba es válida, por lo que estos resultados respaldan los encontrados por la Dra. Nelly Ugarriza quien adapto la prueba a nuestro medio. 
En lo que se refiere al Inventario de habilidades y estrategias de aprendizaje, los análisis psicométricos a los que fue sometido revelan que todos los ítems forman parte de dicho inventario y corresponden a cada una de las diez escalas asignadas previamente. El instrumento es confiable en la medida que sus escalas obtuvieron coeficientes Alfa de Cronbach con valores que oscilan entre 0,69 y 0,78, obteniendo además un alfa de Cronbach total para toda la prueba de 0,91.

En cuanto a la validez, el resultado del Análisis Factorial Exploratorio indica que la prueba está conformada por un solo factor que explica el 69,65\% de la varianza total. Alcanza además un valor de 0,87 en la medida de adecuación del muestreo de Kaiser-Meyer-Olkin, el mismo que puede considerarse como adecuado. Los resultados muestran que la prueba es válida, por lo que estos resultados son coherentes con los encontrados por el Dr. Aníbal Meza Borja, quien adapto la prueba a nuestro medio.

\subsection{Análisis Correlacionales}

Los resultados presentados en la Tabla 1 nos indican que existen correlaciones significativas en todos los casos, a excepción de la escala de Control de Impulsos con ayudas al estudio, notándose que las correlaciones que alcanzan mayor valor son:

- Comprensión de sí mismo $(\mathrm{CM})$ con el total de las estrategias de aprendizaje $(\mathrm{r}=0,61)$ y con procesamiento de la Información $(\mathrm{r}=0,55)$.

- Asertividad (AS) con el total de las estrategias de aprendizaje $(\mathrm{r}=0,63)$ y con Motivación $(\mathrm{r}=0,57)$.

- Autoconcepto (AC) con el total de las estrategias de aprendizaje $(\mathrm{r}=0,68)$ y con Concentración $(\mathrm{r}=0,63)$.

- Autorrealización (AR) con el total de las estrategias de aprendizaje $(r=0,69)$ y con Actitud $(\mathrm{r}=0,62)$.

- Independencia (IN) con el total de las estrategias de aprendizaje ( $\mathrm{r}=0,59)$ y con Concentración e ideas principales $(\mathrm{r}=0,52)$.

- Coeficiente Emocional Intrapersonal (CEIA) con el total de las estrategias de aprendizaje $(\mathrm{r}=0,78)$ y con Motivación $(\mathrm{r}=0,64)$.

- Empatía (EM) con el total de las estrategias de aprendizaje $(\mathrm{r}=0,39)$ y con Ayudas del estudio $(r=0,44)$. 
- Relaciones Interpersonales (RI) con el total de las estrategias de aprendizaje $(r=0,66)$ y con ideas principales $(\mathrm{r}=0,59)$.

- Responsabilidad Social (RS) con el total de las estrategias de aprendizaje ( $\mathrm{r}=0,44)$ y con Autoevaluación $(\mathrm{r}=0,42)$.

- Coeficiente Emocional Interpersonal (CEIE) con el total de las estrategias de aprendizaje $(\mathrm{r}=0,67)$ y con Ayudas del estudio $(\mathrm{r}=0,62)$.

- Solución de Problemas (SP) con el total de las estrategias de aprendizaje ( $r=0,60)$ y con Evaluación $(\mathrm{r}=0,59)$.

- Prueba de la Realidad (PR) con el total de las estrategias de aprendizaje ( $r=0,60)$ y con Gerencia del Tiempo $(\mathrm{r}=0,56)$.

- Flexibilidad (FL) con el total de las estrategias de aprendizaje $(\mathrm{r}=0,61)$ y con Concentración y Evaluación $(\mathrm{r}=0,53)$.

- Coeficiente Emocional Adaptabilidad (CEAD) con el total de las estrategias de aprendizaje $(\mathrm{r}=0,74)$ y con Motivación $(\mathrm{r}=0,64)$.

- Tolerancia al Estrés (TE) con el total de las estrategias de aprendizaje $(r=0,73)$ y con Evaluación $(\mathrm{r}=0,60)$.

- Control de Impulsos (CI) con Gerencia del Tiempo $(\mathrm{r}=0,40)$ y con el total de las estrategias de aprendizaje $(\mathrm{r}=0,38)$.

- Coeficiente Emocional Manejo del estrés (CEME) con el total de las estrategias de aprendizaje $(\mathrm{r}=0,65)$ y con Actitud $(\mathrm{r}=0,58)$.

- Felicidad (FE) con el total de las estrategias de aprendizaje $(\mathrm{r}=0,63)$ y con Actitud $(\mathrm{r}=0,57)$.

- Optimismo $(\mathrm{OP})$ con el total de las estrategias de aprendizaje $(\mathrm{r}=0,73)$ y con Actitud $(\mathrm{r}=0,60)$.

- Coeficiente Emocional estado de ánimo general (CEAG) con el total de las estrategias de aprendizaje $(\mathrm{r}=0,72)$ y con $\operatorname{Actitud}(\mathrm{r}=0,63)$.

- Total Inteligencia Emocional (CE) con el total de las estrategias de aprendizaje $(\mathrm{r}=0,83)$ y con Concentración $(\mathrm{r}=0,66)$. 


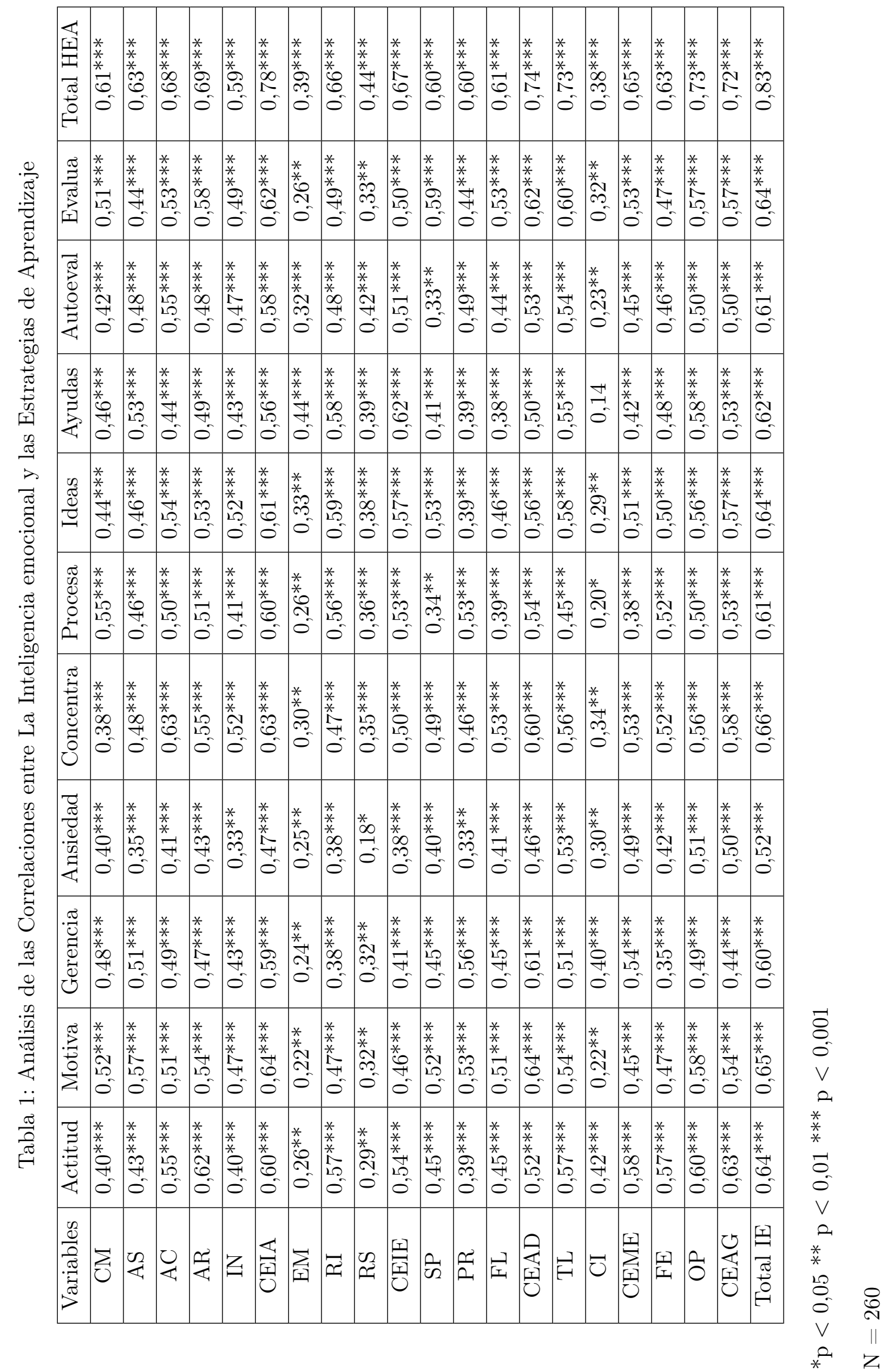




\subsection{Análisis comparativos}

Tabla 2: Prueba Z de comparación de medias de los puntajes de las Escalas de Inteligencia Emocional por Sexo

\begin{tabular}{|c|c|c|c|c|c|c|}
\hline \multirow{2}{*}{ Variable } & \multicolumn{2}{|c|}{ Varón } & \multicolumn{2}{c|}{ Mujer } & \multirow{2}{*}{ N=215 } & \multicolumn{2}{c|}{ N=45. } \\
\cline { 2 - 5 } & $\mathrm{M}$ & $\mathrm{D} . \mathrm{E}$ & $\mathrm{M}$ & $\mathrm{D} . \mathrm{E}$ & & \\
\hline CM & 96,15 & 15,10 & 94,01 & 15,64 & 0,73 & 0,466 \\
\hline AS & 93,71 & 15,57 & 96,68 & 14,27 & $-1,07$ & 0,284 \\
\hline AC & 104,05 & 14,53 & 100,94 & 14,41 & 1,14 & 0,256 \\
\hline AR & 97,21 & 15,08 & 97,10 & 16,04 & 0,03 & 0,971 \\
\hline IN & 100,76 & 12,21 & 100,05 & 17,00 & 0,23 & 0,814 \\
\hline CEIA & 98,21 & 14,98 & 97,18 & 15,95 & 0,34 & 0,731 \\
\hline EM & 80,34 & 16,75 & 82,68 & 15,27 & $-0,79$ & 0,431 \\
\hline RI & 94,50 & 18,42 & 93,09 & 14,83 & 0,47 & 0,639 \\
\hline RS & 82,18 & 14,42 & 84,56 & 14,54 & $-0,86$ & 0,387 \\
\hline CEIE & 87,55 & 17,82 & 87,88 & 14,58 & $-0,11$ & 0,909 \\
\hline SP & 94,92 & 15,21 & 96,10 & 15,43 & $-0,40$ & 0,685 \\
\hline PR & 94,94 & 17,29 & 94,64 & 16,03 & 0,09 & 0,922 \\
\hline FL & 95,97 & 13,25 & 96,38 & 12,94 & $-0,16$ & 0,868 \\
\hline CEAD & 94,47 & 14,69 & 94,64 & 14,77 & $-0,06$ & 0,951 \\
\hline TL & 96,44 & 15,55 & 95,86 & 16,54 & 0,18 & 0,851 \\
\hline CI & 100,39 & 16,79 & 97,91 & 18,55 & 0,72 & 0,470 \\
\hline CEME & 98,36 & 17,26 & 96,36 & 16,47 & 0,63 & 0,526 \\
\hline FE & 97,71 & 16,90 & 94,00 & 15,94 & 1,21 & 0,227 \\
\hline OP & 93,81 & 17,72 & 93,36 & 17,27 & 0,13 & 0,891 \\
\hline CEAG & 94,89 & 18,43 & 92,80 & 17,39 & 0,62 & 0,532 \\
\hline Total IE & 94,05 & 15,87 & 92,99 & 16,32 & 0,34 & 0,729 \\
\hline & & & & & & \\
\hline
\end{tabular}

$* \mathrm{p}<0,05$

$\mathrm{N}=260$

El análisis comparativo de las Escalas de la Inteligencia Emocional por Sexo, indica que no existen diferencias estadísticas significativas en caso alguno, por lo que se puede afirmar que entre los sujetos de la muestra los niveles de inteligencia emocional son similares sin importar su condición de género. 
Tabla 3: Prueba Z de comparación de medias de los puntajes de las Escalas de Estrategias de Aprendizaje por Sexo

\begin{tabular}{|c|c|c|c|c|c|c|}
\hline \multirow[t]{2}{*}{ Variable } & \multicolumn{2}{|c|}{$\begin{array}{l}\text { Varón } \\
\mathrm{N}=215\end{array}$} & \multicolumn{2}{|c|}{$\begin{array}{l}\text { Mujer } \\
\mathrm{N}=45\end{array}$} & \multirow[t]{2}{*}{$\mathrm{Z}$} & \multirow[t]{2}{*}{ Sig. } \\
\hline & $\mathrm{M}$ & D.E & $\mathrm{M}$ & D.E & & \\
\hline Actitud & 28,47 & 4,64 & 29,23 & 4,94 & $-0,82$ & 0,410 \\
\hline Motivación & 28,86 & 3,81 & 29,31 & 4,43 & $-0,55$ & 0,580 \\
\hline Gerencia & 26,36 & 4,40 & 26,13 & 4,98 & 0,26 & 0,795 \\
\hline Ansiedad & 24,34 & 4,37 & 25,06 & 4,73 & $-0,82$ & 0,411 \\
\hline Concentración & 26,84 & 3,50 & 27,40 & 4,70 & $-0,67$ & 0,504 \\
\hline Procesamiento & 30,60 & 4,17 & 29,92 & 4,09 & 0,87 & 0,383 \\
\hline Ideas & 17,44 & 2,96 & 17,29 & 3,12 & 0,25 & 0,799 \\
\hline Ayudas & 26,34 & 5,65 & 27,45 & 5,02 & $-1,13$ & 0,257 \\
\hline Autoevaluación & 28,05 & 5,15 & 27,94 & 4,60 & 0,12 & 0,904 \\
\hline Evaluación & 27,10 & 4,12 & 26,59 & 4,82 & 0,57 & 0,565 \\
\hline Total IHEA & 264,44 & 30,45 & 266,37 & 34,74 & $-0,30$ & 0,762 \\
\hline
\end{tabular}

El análisis comparativo de las Escalas de Estrategias de Aprendizaje por sexo, indica que no existen diferencias estadísticas significativas en caso alguno, por lo que se puede afirmar que entre los sujetos de la muestra las estrategias utilizadas son similares sin importar su condición de género. 
Tabla 4: Análisis de Varianza de un Factor de la Inteligencia Emocional por Ciclo

\begin{tabular}{|c|c|c|c|c|c|c|}
\hline \multirow{2}{*}{ Variable } & $\begin{array}{c}\text { II ciclo } \\
\mathrm{N}=67\end{array}$ & $\begin{array}{c}\text { IV ciclo } \\
\mathrm{N}=75\end{array}$ & $\begin{array}{c}\text { VI ciclo } \\
\mathrm{N}=69\end{array}$ & $\begin{array}{c}\text { VIII ciclo } \\
\mathrm{N}=49\end{array}$ & \multirow{2}{*}{$\mathrm{F}$} & \multirow{2}{*}{ Sig. } \\
\cline { 2 - 5 } & $\mathrm{M}$ & $\mathrm{M}$ & $\mathrm{M}$ & $\mathrm{M}$ & & \\
\hline CM & 96,50 & 91,93 & 96,95 & 94,45 & 0,79 & 0,497 \\
\hline AS & 98,27 & 94,02 & 99,95 & 93,62 & 1,44 & 0,232 \\
\hline AC & 106,00 & 100,08 & 100,80 & 99,87 & 1,62 & 0,187 \\
\hline AR & 102,95 & 94,73 & 96,80 & 94,17 & 2,74 & $0,046^{*}$ \\
\hline IN & 101,80 & 99,26 & 102,25 & 98,77 & 0,40 & 0,752 \\
\hline CEIA & 101,67 & 94,86 & 99,05 & 95,35 & 1,71 & 0,167 \\
\hline EM & 82,37 & 84,91 & 80,35 & 79,42 & 0,95 & 0,414 \\
\hline RI & 95,40 & 92,82 & 93,95 & 92,00 & 0,34 & 0,796 \\
\hline RS & 84,97 & 84,66 & 83,85 & 82,12 & 0,31 & 0,818 \\
\hline CEIE & 90,12 & 88,28 & 86,65 & 85,50 & 0,64 & 0,587 \\
\hline SP & 98,37 & 93,84 & 98,55 & 94,02 & 1,01 & 0,389 \\
\hline PR & 96,87 & 90,97 & 97,15 & 95,57 & 1,21 & 0,308 \\
\hline FL & 97,57 & 94,64 & 97,75 & 96,07 & 0,45 & 0,715 \\
\hline CEAD & 97,00 & 91,62 & 97,25 & 94,22 & 1,20 & 0,312 \\
\hline TL & 98,72 & 93,57 & 98,10 & 95,02 & 0,86 & 0,460 \\
\hline CI & 104,25 & 93,75 & 94,50 & 100,32 & 2,96 & $0,035^{*}$ \\
\hline CEME & 101,72 & 92,66 & 95,90 & 97,30 & 2,17 & 0,094 \\
\hline FE & 98,72 & 93,28 & 96,10 & 92,55 & 1,20 & 0,309 \\
\hline OP & 97,85 & 91,33 & 93,45 & 91,55 & 1,24 & 0,294 \\
\hline CEAG & 98,12 & 91,06 & 94,20 & 90,72 & 1,55 & 0,202 \\
\hline Total IE & 97,22 & 90,84 & 94,05 & 91,65 & 1,29 & 0,279 \\
\hline
\end{tabular}

$* \mathrm{p}<0,05 * * \mathrm{p}<0,01 * * * \mathrm{p}<0,001$

Diferencias significativas por pares de medias a través del test de Scheffe: a, b, c

Los resultados del análisis de varianza de las áreas de la inteligencia emocional por ciclo académico, indican que existen diferencias estadísticas significativas en los siguientes casos:

- Autorrealización (AR) $(\mathrm{F}=2,74 \mathrm{p}<0,05)$; notándose que en el análisis de las comparaciones por pares a posteriori efectuada a través del test de Scheffé, que los alumnos del segundo ciclo $(\mathrm{M}=102,95)$ y del sexto ciclo $(\mathrm{M}=96,80)$ superaron a los alumnos de los otros ciclos.

- Control de Impulsos (CI) $(\mathrm{F}=2,96 \mathrm{p}<0,05)$, notándose que en el análisis de las comparaciones por pares a posteriori efectuada a través del test de Scheffé, que los alumnos del segundo ciclo $(\mathrm{M}=104,25)$ y del octavo ciclo $(\mathrm{M}=100,32)$ superaron a los alumnos del cuarto ciclo $(\mathrm{M}=93,75)$ y a los alumnos sexto ciclo $(\mathrm{M}=94,50)$. 
Tabla 5: Análisis de Varianza de un Factor de las Estrategias de Aprendizaje por Ciclo

\begin{tabular}{|c|c|c|c|c|c|c|}
\hline \multirow{2}{*}{ Variable } & $\begin{array}{c}\text { II ciclo } \\
\mathrm{N}=67\end{array}$ & $\begin{array}{c}\text { IV ciclo } \\
\mathrm{N}=75\end{array}$ & $\begin{array}{c}\text { VI ciclo } \\
\mathrm{N}=69\end{array}$ & $\begin{array}{c}\text { VIII ciclo } \\
\mathrm{N}=49\end{array}$ & \multirow{2}{*}{$\mathrm{F}$} & \multirow{2}{*}{ Sig. } \\
\cline { 2 - 5 } & $\mathrm{M}$ & $\mathrm{M}$ & $\mathrm{M}$ & $\mathrm{M}$ & & \\
\hline Actitud & 30,45 & 28,73 & 28,55 & 28,20 & 1,66 & 0,177 \\
\hline Motivación & 29,02 & 29,06 & 30,35 & 28,95 & 0,56 & 0,642 \\
\hline Gerencia & 26,87 & 26,04 & 25,60 & 25,97 & 0,40 & 0,751 \\
\hline Ansiedad & 25,77 & 24,20 & 24,95 & 24,70 & 0,83 & 0,476 \\
\hline Concentración & 28,20 & 27,00 & 27,20 & 26,62 & 0,93 & 0,427 \\
\hline Procesamiento & 29,95 & 30,04 & 30,80 & 29,97 & 0,22 & 0,881 \\
\hline Ideas & 17,65 & 17,48 & 16,85 & 17,10 & 0,41 & 0,741 \\
\hline Ayudas & 27,05 & 27,64 & 27,90 & 26,37 & 0,57 & 0,636 \\
\hline Autoevaluación & 27,20 & 28,64 & 29,10 & 27,42 & 1,21 & 0,305 \\
\hline Evaluación & 27,60 & 26,66 & 26,25 & 26,17 & 0,72 & 0,536 \\
\hline Total IHEA & 269,77 & 265,53 & 267,55 & 261,50 & 0,41 & 0,739 \\
\hline
\end{tabular}

$* \mathrm{p}<0,05 * * \mathrm{p}<0,01 * * * \mathrm{p}<0,001$

Diferencias significativas por pares de medias a través del test de Scheffe: a, b, c

Los resultados del análisis de varianza de las áreas de las estrategias de aprendizaje por ciclo académico, indican que no existen diferencias estadísticas significativas en caso alguno, por lo que podemos afirmar que los alumnos, sin importar el ciclo académico en el que se encuentren presentan similares niveles de estrategias de aprendizaje.

\section{Conclusiones}

Los resultados logrados nos permiten concluir que existe una relación significativa entre el total de la inteligencia emocional y el total de las estrategias de aprendizaje en los alumnos de la Facultad de Ciencias Matemáticas.

En el análisis de los resultados nos muestra que las diversas áreas de la inteligencia emocional se encuentran relacionadas significativamente con las diversas estrategias de aprendizaje en los alumnos de la Facultad de Ciencias Matemáticas.

En el análisis de las diferencias entre los alumnos varones y mujeres respecto de la prueba de la inteligencia, indica que no existen diferencias estadísticas significativas en caso alguno.

En el análisis de las diferencias entre los alumnos varones y mujeres respecto de las estrategias de aprendizaje, indica que no existen diferencias estadísticas significativas en caso alguno. 
En los resultados del análisis de varianza de las áreas de la inteligencia emocional por ciclo académico, indican que existen diferencias estadísticas significativas en los casos de autorrealización (AR) y control de impulsos (CI), notándose que en el análisis de las comparaciones por pares a posteriori efectuada a través del test de Scheffé, que los alumnos del segundo ciclo superaron a los alumnos de los otros ciclos académicos.

Finalmente, los resultados del análisis de varianza de las áreas de las estrategias de aprendizaje por ciclo académico, indican que no existen diferencias estadísticas significativas en caso alguno, por lo que podemos afirmar que los alumnos, sin importar el ciclo académico en el que se encuentren presentan similares niveles de estrategias de aprendizaje. 


\section{REFERENCIAS BIBLIOGRÁFICAS}

[1] Bara Soro, P. (2001) Estrategias metacognitivas y de aprendizaje: estudio empírico sobre el efecto de la aplicación de un programa metacognitivo, y el dominio de las estrategias de aprendizaje en estudiantesde E.S.O, B.U.P y universidad. (Tesis doctoral). Universidad Complutense de Madrid, España.

[2] Barrios M. (s.f) Las estrategias para fortalecer el aprendizaje en los estudiantes de fundamentos de Enfermería. Recuperado de http://www.portalesmedicos.com/publicaciones/articles/4515/1/.

[3] BarOn, R. (2000). Inventario De Cociente Emocional De BarOn. Lima. Grafimag S.R.L.

[4] Bizquera, R. (2000). Educación emocional y bienestar. Barcelona: Praxis.

[5] Beltrán, J. (1995). Estrategias de aprendizaje. En: Beltrán y Bueno (coord.), Psicología de la Educación (pp. 307 . 329) Madrid: Alianza Editorial.

[6] Goleman, D. (1996). La inteligencia emocional. Primera Edición. Barcelona. Javier Vergara Editor S.A.

[7] Hernández, R., Fernández, C. y Baptista, P. (2006). Metodología de la Investigación. México: Ed. Mc Graw Hill.

[8] Monereo, C., Castelló, M., M. Clariana; Palma, M. y Pérez, Luisa. (2000) Estrategias de enseñanza aprendizaje. Edit. GRAO. Octava edición. Barcelona. España. 